\title{
CAUSALITY AND DISPERSION RELATIONS FOR FIXED MOMENTUM TRANSFER
}

\author{
by H. M. NUSSENZVEIG \\ Instituut voor theoretische fysica der Rijksuniversiteit, Utrecht, Nederland *)
}

\section{Synopsis}

In order to investigate which physical assumptions are relevant to the validity of dispersion relations for fixed momentum transfer, a simple case is treated: the scattering of a classical scalar field by an arbitrary spherically symmetric scatterer of finite radius. It is sufficient to assume: $(a)$ restrictions, due to causality, on the propagation of signals with sharp fronts; $(b)$ conditions on the behaviour of the phase-shifts in the low-frequency and high-angular-momentum limits. To relate the scattering amplitude for fixed momentum transfer with the principle of strict causality, a new representation for this amplitude, in terms of the scattered wave at finite distances from the scatterer, is introduced. The dispersion relations are rigorously derived from the basic assumptions. The results are partially extended to the scattering of Schrödinger particles. An explicit example (totally reflecting sphere) is treated as an illustration.

1. Introduction and survey of results. (a) Relation to previous work. The connection between causality and dispersion relations for the $S$-matrix has been thoroughly investigated by Van Kampen, who has considered the scattering of a classical electromagnetic field ${ }^{1}$ ) or of a Schrödinger particle ${ }^{2}$ ) by a scatterer of finite radius. He has shown that the dispersion relations follow from a very small number of assumptions about the scatterer; the most essential one is a causality condition. In quantum field theory, the dispersion relations have also been derived from a set of general assumptions (although the assumptions concerning the interaction are more specific in this case), and a causality condition also plays an essential role ${ }^{3}$ ).

An entirely different approach has been taken in the derivations of dispersion relations for fixed momentum transfer which have been given by Khuri ${ }^{4}$ ) and by Klein and Zemach ${ }^{5}$ ) for the scattering of Schrödinger particles by a potential, and by Khuri and Treiman ${ }^{6}$ ) for the scattering of Dirac particles by a potential. The form of the interaction is completely specified in these cases, and the derivations are based on the formal solution of the scattering integral equation, by means of a Fredholm expansion ${ }^{4}{ }^{6}$ ) or a Liouville-Neumann expansion ${ }^{5}$ ). It has also been stressed that no explicit use is made of a causality condition. However, it is clear that many

*) On leave of absence from Centro Brasileiro de Pesquisas Físicas, Rio de Janeiro, Brasil 
physical assumptions are implicitly contained in the formal solutions employed in these derivations. The adopted procedure makes it very hard to see which of these assumptions play a significant role. Morenver, it is difficult to predict how the results would be affected in case some of the assumptions were modified. Thus, while this approach may be useful for establishing the domain of applicability of dispersion relations in specific problems, it does not lead to a clear understanding of the physical content of these relations.

In the present paper, following Van Kampen's approach, a simple and rigorous derivation of the dispersion relations for fixed momentum transfer will be given. The derivation, which refers to the simple case of a classical scalar field and a scatterer of finite radius, is based on a small number of physical assumptions. It will be presented in such a way that the assumptions which are relevant to the validity of the results can be recognized at each step. The treatment can be at least partially extended to the case of Schrödinger particles. The results are less general than Khuri's, in the sense that only scatterers of finite radius will be considered; on the other hand, the nature of the interaction need not be specified. The main result which will be obtained is that, contrary to what has been suggested in the literature, the dispersion relations for fixed momentum transfer are closely related with causality.

The remainder of this section is devoted to a heuristic formulation of the basic assumptions and a general survey of the results. The aim is to present a physical picture of the derivation, leaving aside mathematical considerations. Thus, the assumptions will be stated only in very loose terms; precise statements will be given in the subsequent sections.

(b) Preliminary considerations. The scattcring amplitude $f(k, \tau)$, expressed as a function of the wave number $k$ and the momentum transfer $\tau$, is obtained from the scattering amplitude $f(k, \theta)$ in a given direction by making the substitution

$$
\cos \theta=1-\frac{\tau^{2}}{2 k^{2}},
$$

which expresses the relation between the scattering angle and the corresponding momentum transfer. In the cases which we shall consider, the well-known partial-wave expansion of $f(k, \theta)$ can be employed. Replacing (1) in this expansion, we find

$$
f(k, \tau)=\Sigma_{0}^{\infty} f_{l}(k, \tau)=\Sigma_{0}^{\infty} \frac{(2 l+1)}{2 i k}\left[S_{l}(k)-1\right] P_{l}\left(1-\frac{\tau^{2}}{2 k^{2}}\right),
$$

where $S_{l}(k)$ is the $S$-function for the $l$ th partial wave, and $P_{l}$ is the $l$ th Legendre polynomial. According to (1), the physical range of values of $k$ corresponding to a given value of $\tau$ is: $k \geqslant \frac{1}{2} \tau$. We shall assume the validity 
of the usual symmetry relation, $S_{l}(-k)=S_{l}{ }^{*}(k)$, which allows us to define $f(k, \tau)$ for negative $k$ by

$$
f(k, \tau)=f^{\star}(k, \tau) .
$$

The 'non-physical region' is therefore the interval $-\frac{1}{2} \tau<k<\frac{1}{2} \tau$. Equation (2) has been employed by Khuri $\left.{ }^{4}\right)$ to define $f(k, \tau)$ in the non-physical region. In the present paper, the partial-wave expansion will be employed not only on the whole real axis, but also to define the analytic continuation of $f(k, \tau)$ to complex values of $k$. This will allow us to make use of Van Kampen's results on the analytic behaviour of $S_{l}(k)$.

The usual method to derive dispersion relations consists of two steps: (i) proof that $f(k, \tau)$, considered as a function of $k$, for fixed $\tau$, has a regular analytic continuation in the upper half-plane (in the Schrödinger case, however, there may be poles on the positive imaginary axis); (ii) determination of the asymptotic behavior of $f(k, \tau)$ for $|k| \rightarrow \infty$ in the upper halfplane. If $f(k, \tau)$ does not diverge more strongly than a power of $k$, it is possible to derive dispersion relations by carrying out a finite number of 'subtractions'. In the case considered by Khuri, it followed from appropriate restrictions on the potential that $f(k, \tau)$ is bounded at infinity. It must be emphasized that step (ii) is essential in a rigorous derivation.

In sections 2 to 4 , we shall consider a classical scalar field, which obeys the wave equation (we shall take the velocity of propagation $=c=1$ ) in the exterior of a spherically symmetric scatterer of radius $a$. We shall take over Van Kampen's assumptions ${ }^{1}$ ) concerning the interaction. The main results on the analytic behaviour of $S_{l}(k)$ in the complex $k$-plane are quoted below $\left.{ }^{*}\right)$. We shall denote by $I_{0}$ and $I_{+}$the real $k$-axis and the upper half of the $k$-plane, respectively, and by $I_{0+}$ the real axis together with the upper half-plane. The following causality condition was postulated by Van Kampen:

Van Kampen's causality condition (classical field): If an ingoing multipole wave packet vanishes for all time $t<t_{0}$ on a large sphere of radius $r$ surrounding the scatterer, the outgoing wave also vanishes on this sphere for all $t<t_{0}+$ $+2(r-a)$.

The following results, valid for all values of $l$, were derived by Van Ka mpen:

$$
\begin{gathered}
S_{l}(k) \text { is regular in } I_{+}, \\
\left|S_{l}(k) \exp (2 i k a)\right| \leqslant 1 \text { in } I_{+} .
\end{gathered}
$$

These results still do not allow us to draw any conclusion about the analytic behavior of $f(k, \tau)$. It is easy to devise examples of $S_{l}(k)$ such that the partial-wave expansion does not converge for any value of $k$, even though (4) and (5) are satisfied. The point is that the convergence of the partial-

\footnotetext{
*) Van Kampen treated the case of a classical electromagnetic field, but his results can readily be adapted to the present case.
} 
wave expansion imposes restrictions on the behaviour of $S_{l}(k)$ as a function of $l$, whereas no such restriction follows from Van Kampen's results.

(c) The convergence conditions. The nature of the restrictions which are imposed by the convergence of the partial-wave expansion is twofold. In the first place, for any value of $k$, it is necessary that $S_{l}(k)-1$ shall approach zero sufficiently rapidly for $l \rightarrow \infty$. This means that partial waves of sufficiently high angular momentum must be practically unaffected by the scatterer. In the second place, the convergence must be ensured at the critical point $k=0$. This may be seen from (2): the factor $k^{-1} P_{l}\left(1-\tau^{2} / 2 k^{2}\right)$ has a pole of order $2 l+1$ at $k=0$. The simplest way to compensate this singularity is to have a corresponding convergence factor in $S_{l}(k)-1$ for $k \rightarrow 0$. We conclude, therefore, that a convergence condition, prescribing the behaviour of the phase-shifts in the low-frequency and high-angularmomentum limits, is required. A condition which embodies these requirements, the 'weak convergence condition', will be formulated in section 2 . When this condition, which will be postulated only for real values of $k$, is taken in conjunction with Van Kampen's results, it already suffices to prove that the analytic continuation of $f(k, \tau)$, defined by the partial-wave expansion, is regular in $I_{+}$, including the origin.

The only remaining problem is the asymptotic behaviour of $f(k, \tau)$ for $|k| \rightarrow \infty$. An argument based on the optical theorem suggests that $f(k, 0)$ (forward scattering amplitude) cannot diverge more strongly than linearly with $k$ on the real axis. In order to obtain an equivalent limitation for $f(k, \tau)$, we shall employ a more restrictive convergence condition for large values of $k a$. This 'strong convergence condition' will be formulated in section 3 . It requires, essentially, that, for large $k a$, partial waves of sufficiently high angular momentum (such that the corresponding impact parameter is much larger than the radius of the scatterer) shall not be much distorted by the scatterer. It follows from this condition, as will be shown in section 3 , that $f(k, \tau)$ cannot diverge more strongly than linearly with $k$ in $I_{0}$.

(d) The strict causality condition. It is still necessary to consider the asymptotic behaviour of $f(k, \tau)$ in $I_{+}$. A very significant difficulty arises in this connection. According to (5), the asymptotic expansion of $S_{l}(k)$ for large $|k a|$ may contain a factor $\exp (-2 i k a)$. This factor appears, for instance, in the example of a totally reflecting sphere (section 6), and it is also known to appear in the case of Schrödinger particles scattered by a potential of finite radius. The presence of this factor implies that each term of the partial-wave expansion blows up exponentially for $|k| \rightarrow \infty$ in $I_{+}$. Nevertheless, in the case treated by Khuri ${ }^{4}$ ), the asymptotic expansion of $f(k, \tau)$ contains no exponential factor. In order that this shall hold also in the present case, irrespective of the specific form of the interaction, it is clear that some very powerful physical principle is required.

The exponential factor in (5) arises from the phase advancement (corre- 
sponding to a distance $2 a$ ) which takes place when a spherical wave front is reflected at the surface of the scatterer. Let us now consider the effect of such a factor when the scatterer is stricken by an incident wave with a plane wave front. If the phase factor were still present in this case, it would give rise to the immediate appearance of a scattered wave all over the surface of the scatterer. This instantaneous propagation is not ruled out by Van Kampen's causality condition, which refers only to spherical wave fronts. However, it would contradict the principle of strict causality, according to which no signal can be transmitted with a velocity greater than $c$. Arguments of this type have often been employed to show that an exponential factor cannot appear in the particular case of forward scattering $(\tau=0)$.

The question now arises whether the principle of strict causality still enables us to eliminate the exponential factor for $\tau \neq 0$. The answer is affirmative, as will be shown in section 4 . The following condition will be postulated:

Strict causality condition: For an incident wave with a plane wave front, the scattered wave must vanish at any point in space which has not yet been reached by the incident wave.

It is noteworthy that this condition is entirely independent of the radius of the scatterer, and may be applied at any distance from the scatterer. This is closely related with the fact that the radius of the scatterer does not appear in the dispersion relations for $f(k, \tau)$, in contrast with the case of the dispersion relations for $S_{l}(k)$ or $f(k, \theta)(\theta \neq 0)$.

In order to apply the strict causality condition to the determination of the asymptotic behaviour of $f(k, \tau)$ in $I_{+}$, it is necessary to find a relation between $f(k, \tau)$ and the scattered wave. In section 4 , we shall derive from Huygens' principle an integral representation of $f(k, \tau)$ in terms of the scattered wave and its normal derivative, evaluated on a spherical surface around the scatterer. We shall then apply the strict causality condition, to prove that the scattered wave and its normal derivative, regarded as functions of $k$, have a regular analytic continuation in $I_{+}$, and also to determine upper bounds for these quantities in $I_{+}$. This will finally enable us to eliminate the exponential factor, and to prove that $f(k, \tau)$ cannot diverge more strongly than linearly with $k$ in $I_{+}$. The dispersion relations for fixed momentum transfer follow from the results of sections 2 to 4 .

(e) Additional results. Section 5 deals with the extension of the previous results to the case of Schrödinger particles and a scatterer of finite radius. It has been shown by Van Kampen ${ }^{2}$ ) that, in spite of some difficulties, a 'causality condition' can also be formulated in this case, and results similar to (4) and (5) can be obtained. The only essential modification is the possible appearance of poles (usually associated with bound states) on the positive imaginary axis. These results have been derived by Van Kampen only for $s$-waves, but it is very likely that they can be extended 
to higher angular momenta: this will be assumed in section 5 . We shall restrict ourselves to the case in which the total number of poles on the positive imaginary axis is finite. The convergence conditions can be taken over without any change. In the particular case of a potential of finite radius belonging to the class considered by $\mathrm{Khuri}$, all the assumptions are fulfilled. It follows from thesc assumptions that $f(k, \tau)$ is regular in $I_{+}$, except for a finite number of poles on the positive imaginary axis, and cannot diverge more strongly than linearly with $k$ in $I_{0}$. The extension of the results of section 4 seems to be a more difficult problem, and it will not be undertaken here.

Section 6 is devoted to an illustrative example: the case of a totally reflecting sphere. Explicit results, which agree with those obtained in the previous sections, will be given. The problem of the numerical convergence of the partial-wave expansion in the non-physical region, which is of great interest for the practical application of dispersion relations, will be discussed in this example.

2. Weak convergence condition and regularity in $I_{+}$. In this section, as well as in sections 3 and 4 , we shall consider the scattering of a classical scalar field by a spherically symmetric scatterer of radius $a$. We have seen in the introduction that, in addition to Van Kampen's assumptions, we need a condition on the behaviour of $S_{l}(k)-1$ at low frequencies or high angular momenta. In the low-frequency limit, we want to compensate the singularity at the origin arising from the factor $k^{-1} P_{l}\left(1-\tau^{2} / 2 k^{2}\right)$ in (2). For this purpose, it suffices to assume that

$$
S_{l}(k)-1=O\left(k^{2 l+1}\right) \quad \text { for } \quad k \rightarrow 0 .
$$

It follows from (6) that we may define, for each $l$, a range of values of $k$, $0 \leqslant k<k_{l}$, in which $\left|S_{l}(k)-1\right| \leqslant c_{l} k^{2 l+1}<2$, where $c_{l}$ is a constant. It follows, a fortiori, that

$$
\left|S_{l}(k)-1\right| \leqslant 2\left(k / k_{l}\right)^{2 l+1} \quad\left(0 \leqslant k<k_{l}\right) .
$$

Thus, for sufficiently low frequencies $\left(k \ll k_{l}\right)$, the effect of the scatterer on the $l$ th partial wave may be considered as a small perturbation. This will not be true if $k=0$ is the center of a resonance line. However, for $k \neq 0$, resonances are allowed, since no restrictions have been made, as yet, on the behaviour of $k_{l}$.

Now, let us consider the behaviour of $k_{l}$ for $l \rightarrow \infty$. For a scatterer of finite radius, it may be expected that, for any given $k$, partial waves of sufficiently high angular momentum will not be much distorted by the scatterer. Thus, it is reasonable to assume that, for very large $l$, the domain of validity of (7) will increase with $l$, so that

$$
k_{l} \rightarrow \infty \text { for } l \rightarrow \infty .
$$


No assumptions will be made, for the moment, as to the rate of growth of $k_{l}$ with $l$; it may be arbitrarily slow.

The above assumptions can be summed up as follows:

Weak convergence condition: There exists a sequence of wave numbers $k_{l}$ $(l=0,1,2, \ldots)$ such that $(7)$ and $(8)$ are satisfied.

This condition may be considered as an additional restriction on the nature of the scatterer. It will now be shown that the weak convergence condition, together with Van Kampen's results, implies the regularity of $f(k, \tau)$ in $I_{+}$.

According to (4) and (7), $S_{l}(k)-1$ is a regular analytic function in $I_{+}$, with a zero of order (at least) $2 l+1$ at the origin. On the other hand, $P_{l}\left(1-\tau^{2} / 2 k^{2}\right)$ is a polynomial of order $l$ in $\tau^{2} / k^{2}$. It follows that $f_{l}(k, \tau)$, the general term of (2), is a regular analytic function of $k$ in $I_{+}$.

According to Weierstrass's theorem 7), to prove the regularity of $f(k, \tau)$ in any bounded domain of $I_{+}$, it now suffices to show that the partial-wave expansion is uniformly convergent in such a domain. For this purpose, we shall derive a basic inequality for $\left|S_{l}(k)-1\right|$.

Let us notice, to begin with, that, since $\left|S_{l}(k)-1\right| \leqslant 2$ for real $k$, the inequality (7) is certainly valid for $k \geqslant k_{l}$, so that it can be extended to the whole real axis. Thus, if we consider the function

$$
\varphi_{l}(k)=\frac{1}{2}\left(k_{l} / k\right)^{2 l+1}\left[S_{l}(k)-1\right] \exp (2 i k a),
$$

it follows that

$$
\left|\varphi_{l}(k)\right| \leqslant 1 \text { in } I_{0} .
$$

According to (4) and (7), $\varphi_{l}(k)$ is regular in $I_{+}$; also, according to (5), $\left|\psi_{l}(k)\right| \rightarrow 0$ for $|k| \rightarrow \infty$ in $I_{+}$. It may then be concluded from the maximummodulus theorem (reference 7, p. 165) that the inequality $(10)$ is still valid in $I_{+}$. Thus,

$$
\left|S_{l}(k)-1\right| \leqslant 2\left|k / k_{l}\right|^{2 l+1}|\exp (-2 i k a)| \text { in } I_{0+},
$$

which is the basic inequality referred to above.

It follows from the inequality ${ }^{8}$ )

$$
\left|P_{l}(z)\right| \leqslant\left|z \pm\left(z^{2}-1\right)^{1 / 2}\right|^{l},
$$

which is valid for arbitrary (real or complex) $z$, that

$$
\left|P_{l}\left(1-\frac{\tau^{2}}{2 k^{2}}\right)\right| \leqslant\left(3+\left|\frac{\tau^{2}}{k^{2}}\right|\right)^{l} .
$$

Let us restrict $k$ to the semi-circular domain $D_{K}$ of $I_{0+}$ which is defined by: $|k| \leqslant K$. Putting together (2), (11) and (13), we find that

$\left|f_{l}(k, \tau)\right| \leqslant u_{l}(\tau)=(2 l+1)\left|k_{l}\right|^{-2 l-1}\left(3 K^{2}+|\tau|^{2}\right)^{l} \exp (2 K a)$ in $D_{K}$. 
It follows from (8) and (14) that

$$
\lim _{l \rightarrow \infty}\left[\frac{u_{l+1}(\tau)}{u_{l}(\tau)}\right]=\left(3 K^{2}+|\tau|^{2}\right) \lim _{l \rightarrow \infty}\left(\left|k_{l+1}\right|^{-2}\left|\frac{k_{l}}{k_{l+1}}\right|^{2 l+1}\right)=0,
$$

so that the partial-wave expansion converges absolutely and uniformly in $D_{K}$. Since $K$ may be taken arbitrarily large, we finally conclude that the partial-wave expansion is absolutely and uniformly convergent in any bounded domain of $I_{0}$, and $f(k, \tau)$ is a regular analytic function of $k$ in $I_{+}$.

It also follows from the above results that $f(k, \tau)$ is, for fixed $k$ in $I_{0+}$, a regular analytic function of $\tau^{2}$ in the whole complex $\tau^{2}$-plane. In fact, $f_{l}(k, \tau)$ possesses this property, and, if we consider the domain $|\tau|^{2} \leqslant T^{2}$, the inequality (14), with $|\tau|^{2}$ replaced by $T^{2}$, may be applied in this domain, so that the partial-wave expansion is also uniformly convergent with respect to $\tau^{2}$.

3. Strong convergence condition and asymptotic behaviour in $I_{0}$. We must now investigate the asymptotic behaviour of $f(k, \tau)$ for $|k| \rightarrow \infty$. This will be done first for real values of $k$. Throughout this section, it will be assumed that $k a \geqslant k a \gg 1$, where $\bar{k}$ is a sufficiently large wave number; the actual order of magnitude of $\bar{k}$ (which may depend on the range and strength of the interaction) is irrelevant, since we are concerned only with the limit $k \rightarrow \infty$. We shall also introduce the notation: $\beta=k a$.

So far, no assumptions have been made concerning the rate of growth of $k_{l}$ in (8). However, the asymptotic behaviour of $f(k, \tau)$ is clearly related to the rate of convergence of the partial-wave expansion for large $k$, so that we shall now make a more specific assumption.

It is well known that an 'impact parameter' of the order of $l / k$ can be associated with the $l$ th partial wave. If this parameter is much larger than the radius of the scatterer, it is to be expected that the corresponding partial wave will not suffer much distortion. A convenient measure of distortion may be defined as follows: let $\gamma_{l}(k, a)$ be the logarithmic derivative of the radial wave function, evaluated at the surface of the scatterer. Let $\gamma_{l}{ }^{0}(k, a)$ be the value of this quantity in the absence of a scatterer, which is given by

$$
\gamma_{l}{ }^{0}(k, a)=k j_{l}^{\prime}(\beta) / j_{l}(\beta),
$$

where $j_{l}$ is the spherical Bessel function of order $l$. We shall take as a measure of distortion the following quantity:

$$
\delta_{l}(k, a)=\left[\gamma_{l}(k, a)-\gamma_{l}^{0}(k, a)\right] / \gamma_{l}^{0}(k, a) .
$$

On the basis of what has been said above, it may be assumed that this quantity does not exceed unity for $l / k \gg a$, i.e. for $l \geqslant N \beta$, where $N$ is a large number (we shall always take $N \gtrsim 2$ ). This assumption can now be 
formulated as follows:

Strong convergence condition: $\left|\delta_{l}(k, a)\right| \leqslant 1$ for $l \geqslant N \beta$.

A similar condition is usually employed ${ }^{9}$ ) to prove the convergence of the partial-wave expansion for a scatterer of finite radius. In the present case, it will be employed only for large values of $\beta$.

The $S$-function can be expressed in terms of $\delta_{l}(k, a)$ by the following exact formula:

$$
S_{l}(k)-1=-2 i \beta^{2} j_{l}(\beta) j_{l}{ }^{\prime}(\beta) \delta_{l}(k, a)\left[1+i \beta^{2} j_{l}{ }^{\prime}(\beta) h_{l}(\beta) \delta_{l}(k, a)\right]^{-1},
$$

where $h_{l}$ is the spherical Hankel function of the first kind ${ }^{*}$ ) of order $l$.

It follows from (18) and (19) that

$$
\left|S_{l}(k)-1\right| \leqslant 2\left(\frac{e \beta}{2 l+1}\right)^{2 l+1} \text { for } l \geqslant N \beta,
$$

where $e$ is the base of natural logarithms. The derivation of this inequality will be briefly sketched in appendix $\mathrm{A}^{* *}$ ).

Now let us consider the behaviour of the partial-wave expansion for a value of $k$ that satisfies the conditions: $k \geqslant \bar{k}, k \geqslant \frac{1}{2} \tau$, so that $\left|P_{l}\left(1-\tau^{2} / 2 k^{2}\right)\right| \leqslant 1$. It follows from (2) and (20), by employing the majoration $\left|S_{l}(k)-1\right| \leqslant 2$ for $l<N \beta$, that

$$
|f(k, \tau)| \leqslant \frac{1}{k} \sum_{l=0}^{N \beta-1}(2 l+1)+e a \sum_{l=N \beta}^{\infty}\left(\frac{e \beta}{2 l+1}\right)^{2 l} \leqslant(N a)^{2} k+e a \sum_{l=N \beta}^{\infty}\left(\frac{e}{2 N}\right)^{2 l},
$$

or, finally,

$$
|f(k, \tau)| \leqslant(N a)^{2} k+\frac{e a}{1-(e / 2 N)^{2}}\left(\frac{e}{2 N}\right)^{2 N k a} \quad\left(k \geqslant \bar{k}, k \geqslant \frac{1}{2} \tau\right) .
$$

Notice that, according to the optical theorem, (21) implies that the total cross-section in the high-frequency limit cannot exceed $4 \pi(N a)^{2}$.

It follows from (21) and from the symmetry relation (3) that

$$
|f(k, \tau)|=O(|k|) \text { for }|k| \rightarrow \infty \text { in } I_{0} .
$$

An explicit example in which a linear divergence occurs will be given in section 6 .

The only remaining problem is the asymptotic behaviour of $f(k, \tau)$ in $I_{+}$. It may be seen at once that (11) is of no avail in this respect, owing to the factor $|\exp (-2 i k a)|$, which blows up exponentially in $I_{+}$. This difficulty has been discussed in the introduction, and it has been stated that the ex-

*) Since only spherical Hankel functions of the first kind will occur in the present work, we shall omit the superscript (1).

**) Notice that, if one assumes the validity of the strong convergence condition for $0 \leq \beta \leq l / N$ (for large $l$ ), it follows from (20) that one may take in (7) $k_{l}=l !(\mathrm{Na}$ ) (for large $l$ ). 
ponential factor can be eliminated by applying the strict causality condition. This will be proved in the next section.

4. Strict causality and asymptotic behaviour in $I_{+}$. We shall start by deriving an integral representation of $f(k, \tau)$. Let the total wave function at the point $\boldsymbol{R}=(R, \theta, \varphi)$ in the exterior of the scatterer, corresponding to an incident plane wave $\exp [i k(R \cos \theta-t)]$, be given by

$$
\varphi(k, \boldsymbol{R}, t)-\left[\exp (i k R \cos \theta)+u_{s}(k, R, \theta)\right] \operatorname{cxp}(-i k t) .
$$

According to Huygens' principle 10 ), the scattered wave $u_{s}(k, \boldsymbol{R})$ can be represented in the following form:

$$
u_{s}(k, \boldsymbol{R})=\oint_{\boldsymbol{\sigma}}\left[u_{s}(k, \boldsymbol{r}) \frac{\partial G}{\partial n}(k, \boldsymbol{R}, \boldsymbol{r})-G(k, \boldsymbol{R}, \boldsymbol{r}) \frac{\partial u_{s}}{\partial n}(k, \boldsymbol{r})\right] \mathrm{d} \sigma,
$$

where $n$ denotes the direction of the outward normal to the closed surface $\sigma$ surrounding the scatterer, and

$$
G(k, \boldsymbol{R}, \boldsymbol{r})=\frac{\exp (i k|\boldsymbol{R}-\boldsymbol{r}|)}{4 \pi|\boldsymbol{R}-\boldsymbol{r}|} .
$$

We shall choose $\sigma$ as a sphere of radius $r=|\boldsymbol{r}| \geqslant a$, concentric with the scatterer.

If we now let $R \rightarrow \infty$ along the direction $R / R$, we find from (24) the following expression for the scattering amplitude in this direction:

$$
f(k, \theta)=-\frac{r^{2}}{4 \pi} \oint_{\sigma}\left[i \frac{\boldsymbol{k} \cdot \boldsymbol{r}}{\boldsymbol{r}} u_{s}(k, \boldsymbol{r})+\frac{\partial u_{s}}{\partial r}(k, \boldsymbol{r})\right] \exp (-i \boldsymbol{k} \cdot \boldsymbol{r}) \mathrm{d} \sigma,
$$

where $\boldsymbol{k}=k \boldsymbol{R} / R=(k, \theta, \varphi)$. Let $\boldsymbol{r}=\left(r, \theta^{\prime}, \varphi^{\prime}\right)$; then, the integration with respect to $\varphi^{\prime}$ in (26) can be performed, with the help of the well-known formula

$$
\int_{0}^{2 \pi} \exp \left[i v \cos \left(\varphi-\varphi^{\prime}\right)\right] \mathrm{d} \varphi^{\prime}=2 \pi J_{0}(v),
$$

where $J_{0}$ denotes Bessel's function of order zero. By carrying out the integration and by expressing $\theta$ in terms of $\tau$ with the help of (1), we finally arrive at the following integral representation of $f(k, \tau)$ (the integration variable is now denoted by $\theta$ instead of $\theta^{\prime}$ ):

$$
\begin{gathered}
f(k, \tau)=-\frac{1}{2} r^{2} \int_{0}^{\pi}\left[g_{1}(k, \tau, r, \theta) u_{s}(k, r, \theta)+g_{2}(k, \tau, r, \theta) \frac{\partial u_{s}}{\partial r}(k, r, \theta)\right] . \\
\cdot \exp \left[-i k r \cos \theta\left(1-\frac{\tau^{2}}{2 k^{2}}\right)\right] \sin \theta \mathrm{d} \theta
\end{gathered}
$$

where $r \geqslant a$ is arbitrary, and

$$
\begin{gathered}
g_{1}(k, \tau, r, \theta)=i k \cos \theta\left(1-\frac{\tau^{2}}{2 k^{2}}\right) J_{0}(\tau \zeta r \sin \theta)+\tau \zeta \sin \theta J_{1}(\tau \zeta r \sin \theta), \\
g_{2}(k, \tau, r, \theta)=J_{0}(\tau \zeta r \sin \theta),
\end{gathered}
$$


where $J_{1}$ is Bessel's function of order one, and

$$
\zeta=\left[1-\frac{1}{4}(\tau / k)^{2}\right]^{1 / 2} \text {. }
$$

Notice that the sign of the square root in $(30)$ is irrelevant.

We shall now take (27) to define the analytic continuation of $f(k, \tau)$ to complex values of $k$. It is possible to verify directly that (27) is equivalent to (2), by making the substitution

$$
u_{s}(k, r, \theta)=\frac{1}{2} \sum_{0}^{\infty}(2 l+1) i l\left[S_{l}(k)-1\right] h_{l}(k r) P_{l}(\cos \theta),
$$

and by employing some formulae which may be derived from Gegenbauer's integral 11).

In order to apply the strict causality condition, we shall now take an incident wave packet,

$$
\psi_{0}(r, \theta, t)=\int_{-\infty}^{+\infty} A(k) \exp [-i k(t-r \cos \theta)] \mathrm{d} k .
$$

According to (23), the corresponding scattered wave packet is

$$
\psi_{s}(r, \theta, t)=\int_{-\infty}^{+\infty} A(k) u_{s}(k, r, \theta) \exp (-i k t) \mathrm{d} k .
$$

The strict causality condition, which has been stated in section $1(d)$, can now be formulated as follows:

Strict causality condition: If $\psi_{0}$ vanishes for $t<r \cos \theta$, so does $\psi_{s}$.

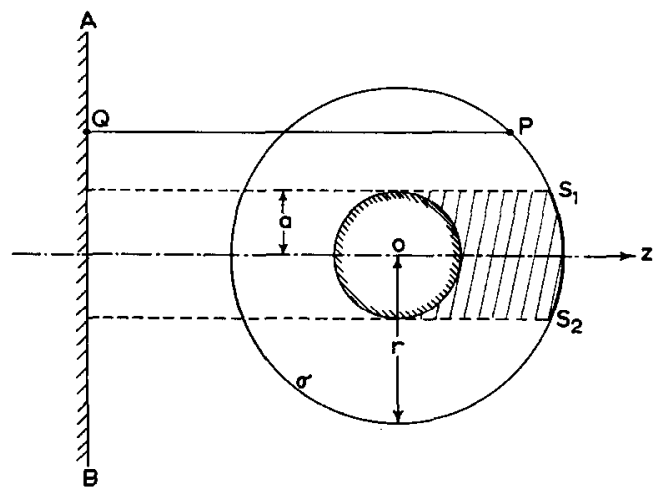

Fig. 1. For an incident wave with a plane wave front $A B$, the scattered wave cannot arrive at any point $P$ before the incident wave arrives there. If $P$ belongs to the geometrical shadow region $S_{1} S_{2}$, no stronger condition can be formulated.

This condition is illustrated in fig. 1, which shows the incident wave front $A B$ at a given moment and a sphere $\sigma$ of radius $r$ surrounding the scatterer. The scattered wave cannot reach any point on $\sigma$ in advance of the incident wave.

It may be seen at once in fig. 1 that causality actually leads to a condition which is stronger than (34) on a large part of $\sigma$. The shortest path connecting a 
point such as $P$ in fig. 1 with the wave front, via the scatterer, is longer than $\overline{P Q}$. However, no condition stronger than (34) can be formulated for those points of $\sigma$ that lie in the geometrical shadow of the scatterer (indicated by $S_{1} S_{2}$ in fig. 1). Thus, (34) is the strongest condition that can be uniformly applied to all the points of $\sigma$. Other advantages of (34) are its simplicity and its independence of the radius of the scatterer. Notice also that, in contrast with Van Kampen's causality condition, (34) ensures the causal propagation of signals across the scatterer.

The aim of the following derivation will be to find upper bounds for $\left|u_{s}\right|$ and $\left|\partial u_{s} / \partial \gamma\right|$ for large $|k|$ in $I_{+}$, so as to obtain from (27) the asymptotic behaviour of $f(k, \tau)$. For this purpose, we shall apply the strict causality condition not only to $\psi_{s}$, but also to $\partial \psi_{s} / \partial r$. It is clear that, in (34), $\partial \psi_{s} / \partial r$ must also vanish for $t<r \cos \theta$.

In order to apply a reasoning similar to that which was employed by Van $\mathrm{Kampen}{ }^{1}$ ) in the derivation of (4) and (5), the integrand of (33), as well as its partial derivative with respect to $r$, must be square integrable. Thus, it is necessary to find bounds for the asymptotic behaviour of $u_{s}$ and $\partial u_{s} / \partial r$ for $k \rightarrow \infty$. For this purpose, we shall employ (31) and the inequality (20).

The value of $r$ in (27), as well as in (34), has been left undetermined hitherto. It is convenient at this point to make, once and for all, the following choice *):

$$
r=N a .
$$

The following inequalities, which are valid for sufficiently large $k(k \geqslant \bar{k})$, can be derived from (20) and (31):

$$
\begin{aligned}
\left|u_{s}(k, N a, \theta)\right| & \leqslant \frac{4}{3} N a k+O\left(\beta^{1 / 2}\right), \\
\left|\frac{\partial u_{s}}{\partial r}(k, N a, \theta)\right| & \leqslant N a k^{2}+O\left(\beta^{3 / 2}\right) .
\end{aligned}
$$

The proof of these results will be given in appendix B. It follows from (36) and (37) that there must exist constants $M$ and $\gamma>0$ such that

$$
\begin{gathered}
\left|u_{s}(k, N a, \theta)\right| \leqslant M|k+i \gamma| \quad \text { if } \quad k \text { is in } I_{0}, \\
\left|\frac{\partial u_{s}}{\partial r}(k, N a, \theta)\right| \leqslant M|k+i \gamma|^{2} \quad \text { if } \quad k \text { is in } I_{0} .
\end{gathered}
$$

It is now possible to choose $A(k)$ in such a way that the following conditions shall be fulfilled: (a) $\psi_{0}$ vanishes for $t<r \cos \theta$; (b) (32) and (33) are square integrable; (c) (33) may be differentiated with respect to $r$ under the integral sign,

$$
\frac{\partial \psi_{s}}{\partial r}=\int_{-\infty}^{+\infty} A(k) \frac{\partial u_{s}}{\partial r}(k, r, \theta) \exp (-i k t) \mathrm{d} k ;
$$

*) The reason for this choice is the rapid increase of $\left|h_{l}(k r)\right|$ for $l>k r$; the choice (35) enables us to make full use of $(20)$ in order to compensate this growth. 
(d) (40) is square integrable. According to (38) and (39), it suffices, for this purpose, to choose ${ }^{*}$ )

$$
A(k)=A_{0}(k+i \gamma)^{-4},
$$

where $A_{0}$ is a constant factor having the appropriate dimensionality.

Notice that it is not sufficient to require that the total incident energy per unit area should be finite, since this is equivalent to the square integrability of $k A(k)$, which is not enough. It also does not seem to be possible to derive stronger limitations than (38) and (39) from physical requirements on the scattered energy. Such requirements usually lead to restrictions on the behaviour of integrals containing the quantities in question, but not on their local behaviour. The singular behaviour of (38) and (39) in the 'geometrical optics limit' $(k \rightarrow \infty)$ is physically related to the possibility of existence of focal points or lines.

The strict causality condition requires that, with the choice (41), the second members of (33) and (40) shall vanish for $t<r \cos \theta$. By applying a familiar reasoning ${ }^{1}$ ), which need not be reproduced here, this leads to the following conclusions $\left.{ }^{* *}\right)$ :

$u_{s}(k, N a, \theta)$ and $\frac{\partial u_{s}}{\partial r}(k, N a, \theta)$ have regular analytic continuations in $I_{+},(42)$

$$
\begin{gathered}
\mid u_{s}(k, N a, \theta)\left(\exp (-i N k a \cos \theta)|\leqslant M| k+i \gamma \mid \text { in } I_{+},\right. \\
\left|\frac{\partial u_{s}}{\partial r}(k, N a, \theta) \exp (-i N k a \cos \theta)\right| \leqslant M|k+i \gamma|^{2} \text { in } I_{+} .
\end{gathered}
$$

The inequalities (43) and (44) are the desired results, which allow us to obtain upper bounds for the asymptotic behaviour of $f(k, \tau)$ in $I_{+}$. If we replace them in (27) (with $r=N a$ ), and if we employ the inequalities (reference 11, p. 49)

$$
\left|J_{0}(z)\right| \leqslant \exp (\operatorname{Im} z),\left|J_{1}(z)\right| \leqslant \frac{1}{2}|z| \exp (\operatorname{Im} z),
$$

to derive upper bounds for (28) and (29), we finally arrive at the following result:

$$
\begin{gathered}
|f(k, \tau)| \leqslant M(N a)^{2}|k+i \gamma|\left[|k+i \gamma|+|k|+\left|\frac{\tau^{2}}{2 k}\right|+\frac{1}{2} N a \tau^{2}\left(1+\left|\frac{\tau}{2 k}\right|^{2}\right)\right] . \\
\cdot \exp \left\{N \tau a\left[\left|\frac{\tau}{2 k}\right|+\left(1+\left|\frac{\tau}{2 k}\right|^{2}\right)^{1 / 2}\right]\right\} \quad \text { in } I_{+} .
\end{gathered}
$$

It follows from (45) that

$$
|f(k, \tau)|=O\left(|k|^{2}\right) \text { for }|k| \rightarrow \infty \text { in } I_{+},
$$

*) It can be verified that, with this choice, (40) is uniformly convergent in an interval around $r=N a$, so that (c) is valid.

**) In the derivation of (43) and (44), the Phragmén-Lindelöf theorem 12) is employed, in conjunction with (38) and (39). 
so that the troublesome exponential factor is indeed eliminated by the strict causality condition.

Let us now notice that, according to (21) and (22), there must exist constants $C$ and $\delta>0$ such that $|f(k, \tau)| \leqslant C|k+i \delta|$ in $I_{0}$. On the other hand, it follows from section 2 that $f(k, \tau) /(k+i \delta)$ is regular in $I_{+}$. It can therefore be concluded from (46) and from the Phragmén-Lindelöf theorem 12 that the above inequality is also valid in $I_{+}$:

$$
|f(k, \tau)| \leqslant C|k+i \delta| \text { in } I_{0+\cdot}
$$

Thus, $f(k, \tau)$ cannot diverge more strongly than linearly with $k$ in $I_{0+}$.

It is worthwhile to point out that the strict causality condition, together with (38) and (39), enables us to give another derivation of the regularity of $f(k, \tau)$ in $I_{+}$, provided that we exclude a neighbourhood of the origin. Let us consider the domain $D_{\varepsilon, K}$ of $I_{0+}$, which is defined by: $0<\varepsilon \leqslant|k| \leqslant K$. If we replace $|k|$ by $\varepsilon$ in the denominators of (45), and by $K$ in the numerators, we obtain an upper bound, independent of $k$ and $\theta$, for the integrand of (27), in $D_{\varepsilon, K}$. According to (28)-(30), $g_{1}$ and $g_{2}$ are regular analytic functions of $k$ in $D_{\varepsilon, K}$. Taking into account (42), we conclude that the integrand of (27) is a regular, uniformly bounded, analytic function of $k$ in $D_{\varepsilon, K}$. It follows from this $\left.{ }^{13}\right)$ that $f(k, \tau)$ is regular in $D_{\varepsilon, K}$. Since $K$ may be taken arbitrarily large and $\varepsilon$ arbitrarily small, we obtain indeed the announced result. However, on account of the denominators in (45), this method does not allow us to prove the regularity at the origin (in contrast with the method of section 2).

We are now in possession of all the results required for the derivation of dispersion relations. It follows from (3), (47), and the results of section 2 , that $f(k, \tau)$ satisfies the following dispersion relation:

$$
\operatorname{Re} f(k, \tau)=f(0, \tau)+\frac{2}{\pi} k^{2} P \int_{0}^{\infty} \frac{\operatorname{Im} f\left(k^{\prime}, \tau\right)}{k^{\prime}\left(k^{\prime 2}-k^{2}\right)} \mathrm{d} k^{\prime},
$$

where $P$ denotes Cauchy's principal value.

5. Extension to Schrödinger particles. (a) General scatterer. In this section, we shall deal with the problem of extending the above treatment to the case of Schrödinger particles. The results which will be presented are incomplete: they rely upon an assumption concerning the possibility of extending Van Kampen's work, and the extension of the results of section 4 will not be considered. Nevertheless, it seems worthwhile to indicate a possible procedure for extending the treatment given in sections 2 and 3 to this case.

We shall consider the scattering of Schrödinger particles by a spherically symmetric scatterer of radius $a$. This problem has been investigated in reference 2 in the case of $s$-waves. The causalit y condition for a classical field 
cannot be applied to Schrödinger particles, owing to several difficulties, so that it was reformulated as follows:

Van Kampen's 'causalily condition' (Schrödinger particles): If an ingoing wave packet is so normalised as to represent at $t=-\infty$ one incident particle, the total probability, at any given moment, of finding a particle outside of the scatterer, cannot be greater than one.

If one postulates, in addition, the usual symmetry relation of the $S$ function, it follows that $S(k)$ cannot have any singularities in $I_{+}$, except on the imaginary axis. If one assumes that there are no other than isolated singularities, there can only be simple poles. It is very likely that these results can be extended to higher order multipole waves. It will be assumed here that Van Kampen's results are valid for all values of $l$.

We shall now restrict our consideration to the case in which the following condition is fulfilled:

The total number of poles of the S-matrix on the positive imaginary axis is finite.

In the problem of scattering by a potential, this is the only case which has been considered hitherto $\left.{ }^{4}\right)^{5}$ ). It follows from (49) that there must exist a finite integer $l_{0}$, such that $S_{l}(k)$ has no poles on the positive imaginary axis for $l \geqslant l_{0}$.

The following conclusions can be derived from the above assumptions: (I) For $l<l_{0}, S_{l}(k)$ is regular in $I_{+}$, except for a finite number of simple poles on the imaginary axis. (II) For $l \geqslant l_{0}$, (4) and (5) remain valid.

It is now a trivial matter to extend the results of sections 2 and 3 to the present case. The weak and strong convergence conditions can be taken over without any modification. If one decomposes the partial-wave expansion into $f_{\mathrm{I}}(k, \tau)$ (the sum from $l=0$ to $l=l_{0}-1$ ) and $f_{\mathrm{II}}(k, \tau)$ (the sum from $l_{0}$ to $\left.\infty\right)$, it follows from $(\mathrm{I})$ that $f_{\mathbf{I}}(k, \tau)$ is regular in $I_{+}$, except for a finite number of poles on the imaginary axis, while, according to (II), the results of section 2 still apply to $t_{\mathrm{II}}(k, \tau)$. No change is necessary in section 3 , since it refers only to real values of $k$. Therefore, according to the above assumptions, the only modification in the results of sections 2 and 3 is that $f(k, \tau)$ may have a finite number of poles on the positive imaginary axis.

Although the integral representation (27) remains valid in the present case, the extension of the results of section 4 seems to be a more difficult problem (the difficulties are of the same nature as those which were discussed in reference 2). This problem will not be considered here.

(b) Special case: potential of finite radius. Now let us consider the special case in which the scatterer is a central potential $V(r)$ (vanishing for $r>a$ ). In order to satisfy condition (49), it is sufficient to impose the restriction ${ }^{14}$ )

$$
\int_{0}^{a} r|V(r)| \mathrm{d} r \leqslant B<\infty,
$$


which is the form taken by Khuri's conditions ${ }^{4}$ ) in the present case. Van Kampen's causality condition and the symmetry relation of the $S$-matrix are always satisfied in this case. The above conclusions (I) and (II) can also be derived independently ${ }^{15}$ ).

Let us discuss the validity of the convergence conditions. It is well known ${ }^{16}$ ) that condition (6), and consequently also (7), is satisfied ${ }^{*}$ ). To prove (8), it suffices to show that, given a wave number $K$ (no matter how large), it is always possible to find an integer $L$ such that

$$
\left|S_{l}(k)-1\right| \leqslant 2(k / K)^{2 l+1} \text { for } 0 \leqslant k<K, \quad l \geqslant L .
$$

To show this, we employ a result due to $C$ arter 17 ), according to which, for any given $k$, and large enough $l, S_{l}(k)-1$ is always bounded by Born's approximation, i.e.

$$
\left|S_{l}(k)-1\right| \leqslant C k \int_{0}^{a} r^{2}|V(r)| j_{l}^{2}(k r) \mathrm{d} r,
$$

where $C$ is a constant. On the other hand, it follows from (50) and from Watson's inequality (reference 11, p. 255) that

$C k \int_{0}^{a} r^{2}|V(r)| j_{l}^{2}(k r) \mathrm{d} r \leqslant \frac{B C}{(2 l+1)}\left(\frac{e k a}{2 l+1}\right)^{2 l+1}$ if $0 \leqslant k a \leqslant \frac{1}{2} l$.

Let $L$ be chosen in such a way that (52) is valid for $0 \leqslant k<K, l \geqslant L$, and also: $L \geqslant 2 K a, 2 L+1 \geqslant \frac{1}{2} B C$. Then, it follows from (52) and (53) that (51) is valid. This shows that $k_{l}$ in (7) cannot approach a finite limit for $l \rightarrow \infty$, so that (8) must be true. Therefore, the weak convergence condition is verified. The validity of the strong convergence condition follows from the validity (at least in order of magnitude) of Born's approximation for $l \geqslant k a$ and sufficiently large $k a{ }^{18}$ ).

Thus, all the assumptions which have been made above are valid in the present case. The results which have been derived from these assumptions agree with those obtained by Khuri ${ }^{4}$ ), with only one exception: we have found no branch points of $f(k, \tau)$ at $k= \pm \frac{1}{2} \tau$. Khuri's claim that such branch points exist, and that they arise from the integral in his equation (20), is incorrect: the integral has no branch points, because it does not depend on the direction of $\boldsymbol{n}$. Our limitation (22) is actually too weak in the present case : it has been shown by Khuri that $f(k, \tau)$ is bounded (and tends to Born's approximation) for $|k| \rightarrow \infty$.

6. An example: totally reflecting sphere. To illustrate the results derived in the previous sections, we shall now consider a special example, namely, scattering by a totally reflecting sphere. The corresponding boundary condition will be taken to be the vanishing of the total wave function on

*) The case in which there exists a 'bound statc at zero encrgy' must be excepted. 
the surface of the sphere. There is no difference between the results for a classical field and those for a Schrödinger particle in this case. This example does not belong to the class of scatterers admitted in previous investigations $\left.{ }^{4}\right)^{5}$ ), but it falls within the scope of the present treatment.

Employing the well-known expression for the phase-shifts corresponding to this example, and the notation $\beta=k a$, we find

$$
S_{l}(k)-1=-2 j_{l}(\beta) / h_{l}(\beta) .
$$

It follows from (54), by employing the expansions of the spherical functions around the origin, that, for real or complex $\beta$,

$$
\begin{gathered}
S_{0}(k)-1=-2 i \beta[1+O(|\beta|)] \quad(|\beta| \ll 1), \\
S_{l}(k)-1= \\
=-\frac{2 i \beta^{2 l+1}}{(2 l-1) ! !(2 l+1) ! !}\left[\frac{1+\theta_{l}(\beta)}{1+\theta_{l}^{\prime}(\beta)}+\frac{i \beta^{2 l+1}}{(2 l-1) ! !(2 l+1) ! !}\right]^{-1}(l \geqslant 1),
\end{gathered}
$$

where $(2 l-1) ! !=1.3 .5 \ldots(2 l-1)$, and, according to Nielsen's inequality (reference 11, p. 44),

$$
\left|\theta_{l}(\beta)\right| \leqslant \frac{1}{(2 l-1)}\left[\exp \left(\frac{1}{2}|\beta|^{2}\right)-1\right] ;\left|\theta_{l}^{\prime}(\beta)\right| \leqslant \frac{1}{(2 l+3)}\left[\exp \left(\frac{1}{2}|\beta|^{2}\right)-1\right] .
$$

Let $l^{\prime}$ be an integer, such that $l^{\prime} \gg 1$ and $\left(\log l^{\prime}\right)^{1 / 2} \gg 1$, and let us consider the behaviour of $S_{l}(k)-1$ for real $k$ and $l \geqslant l^{\prime}$. If $0 \leqslant \beta \leqslant(\log l)^{1 / 2}$, (56) may be employed (with Stirling's approximation for the factorials), and it follows from (57) that the expression within square brackets in (56) differs little from unity. On the other hand, if $1 \ll \beta \leqslant \frac{1}{2} l$, the spherical Bessel and Hankel functions in (54) may be replaced by the Debye asymptotic expansions ${ }^{19}$ ). In this way it is found that

$$
\left|S_{l}(k)-1\right| \leqslant 2\left(\frac{e \beta}{2 l+1}\right)^{2 l+1} \text { for } 0 \leqslant \beta \leqslant \frac{1}{2} l, \quad l \geqslant l^{\prime} .
$$

It follows from (55) to (58) that conditions (6) to (8) are satisfied (with $k_{l}=\frac{1}{2} l / a$ for $\left.l \geqslant l^{\prime}\right)$. According to (58), the inequality (20) is also satisfied (with $N=2)$, in spite of the fact that (18) is not fulfilled $\left(\gamma_{l}(k, a)\right.$ diverges). Therefore, all the results of sections 2 to 4 are valid, and $f(k, \tau)$ satisfies the dispersion relation (48).

In order to apply the dispersion relation in practice, it is necessary to evaluate $f(k, \tau)$ in the non-physical region. The partial-wave expansion can be employed for this purpose only if it converges rapidly enough. It is therefore important to investigate the numerical convergence of (2) in the non-physical region.

It follows from (55) to (57) that the partial-wave expansion converges uniformly in a complex neighbourhood of the origin. Therefore, $f(0, \tau)$ can Physica 26 
be evaluated by taking the limit as $k \rightarrow 0$ under the summation sign, with the following result (which is well known for $\tau=0$ ):

$$
f(0, \tau)=\Sigma_{0}^{\infty} f_{l}(0, \tau)=a \Sigma_{0}^{\infty}(-1)^{l+1} \frac{(\tau a)^{2 l}}{(2 l) !}=-a \cos (\tau a) .
$$

This gives the value of the constant term in (48) for the present example. Moreover, it can be concluded from (59) that the numerical convergence of the partial-wave expansion at the point $k=0$ is rapid for $\tau a \lesssim 1$, but becomes slow for $\tau a \gg 1$. This conclusion can be extended without difficulty to the whole non-physical region.

It is also possible, in this example, to derive an explicit expression for the asymptotic behaviour of $f(k, \tau)$ on the real axis. We shall only quote the result, which is found to be

$$
f(k, \tau) \approx i k a^{2} \frac{J_{1}(\tau a)}{\tau a}+O\left[(k a)^{1 / 3}\right] \quad(k \rightarrow \infty),
$$

where $J_{1}$ denotes Bessel's function of the first order. This result is well known ${ }^{20}$ ) for $\tau=0$. The physical origin of the linear divergence in this case is the diffraction peak in the forward direction ('shadow scattering'). Equation (60) is consistent with (22), and shows that it is not possible to decrease the number of subtractions in the dispersion relation (48) without imposing further restrictions upon the scatterer.

We also mention, without giving the proof, that the partial-wave expansion enables us to extend the definition of $f(k, \tau)$ to the lower half of the $k$-plane, in the present example. It then becomes a meromorphic function of $k$ (no branch points!), having an infinite number of poles in the lower half-plane; these are obtained by taking the poles of $S_{l}(k)$ for all values of $l$.

It may finally be observed that, in agreement with the result obtained at the end of section 2, the last member of (59) is a regular analytic function of $\tau^{2}$ (notice, however, that it has an essential singularity for $\left.\mid \tau^{2}\right\} \rightarrow \infty$ ).

7. Conclusion. The existence of a close connection between causality and dispersion relations for fixed momentum transfer, in the case of a classical field, can now be regarded as clearly established. In the case of Schrödinger particles, the above results, though less complete, indicate that Van Kampen's causality condition plays a significant role. However, the extension of the results of section 4 to this case remains an open problem.

The main requirements which have been employed in the derivation, in addition to causality, are the weak and strong convergence conditions. These conditions are very natural from the physical point of view: they are related to the usual assumption, in phase-shift analysis, that contributions from sufficiently high angular momenta may be neglected. It has been pointed out by Allcock ${ }^{21}$ ) that assumptions of this kind are necessary, 
in order that the analytic continuation of $f(k, \tau)$ by means of the partial-wave expansion shall be stable, i.e. that it shall not depend too critically on the unavoidable uncertainties which affect the experimental data. Since no other method is available for computing $f(k, \tau)$ in the non-physical region, it is clear that such stability is an essential requirement for the practical application of the dispersion relations.

It would be interesting to know to what extent the form of the interaction is restricted by the assumptions made in the present work. While these assumptions can probably be considerably relaxed, without affecting the essential results, it is to be expected that they are valid for a large class of scatterers.

The author is indebted to Professor N. G. Van Kampen for suggesting this problem and for valuable discussions, as well as for a critical reading of the manuscript. He wishes to thank Professor L. Van Hove for the hospitality of the Instituut voor Theoretische Fysica, Utrecht, and to acknowledge his indebtedness to the National Research Council of Brazil for award of a fellowship.

\section{APPENDIX}

(A). Proof of the inequality (20). In this appendix, the derivation of the inequality (20) of the text will be briefly indicated. It will be assumed throughout that $l / N \geqslant k a \geqslant k a \geqslant 1$. Under these conditions, it follows from (18) and (19) that

$$
\left|S_{l}(k)-1\right| \leqslant 2 \beta^{2}\left|j_{l}(\beta) j_{l}(\beta)\right|\left[1-\beta^{2}\left|j_{l}(\beta) h_{l}(\beta)\right|\right]^{-1},
$$

provided that the expression within square brackets is positive.

It follows from Watson's inequalities for $J_{\nu}(\nu x)$ and $J_{\nu}{ }^{\prime}(v x)$ (reference 11, p. 255) that

$$
\beta^{2}\left|j_{l}(\beta) j_{l}(\beta)\right| \leqslant \frac{1}{2}\left(\frac{e \beta}{2 l+1}\right)^{2 l+1} .
$$

The expression within square brackets in (A 1) can be estimated by employing Debye's asymptotic expansions ${ }^{19}$ ). It is found that, under the above conditions,

$$
\beta^{2}\left|i_{l}^{\prime}(\beta) h_{l}(\beta)\right| \leqslant \frac{1}{2} .
$$

Substituting (A 3) and (A 2) in (A 1), one obtains the inequality (20) of the text. An equivalent inequality has been proved by Schiff ${ }^{9}$ ) for $l \gg \beta^{2}$.

(B) The inequalities (36) and (37). To prove the inequality (36) of the text, we notice first that, according to (31),

$$
\left|u_{s}(k, N a, \theta)\right| \leqslant \frac{1}{2} \sum_{0}^{\infty}(2 l+1)\left|S_{l}(k)-1\right|\left|h_{l}(N \beta)\right|,
$$


where $\beta=k a$. To estimate $\left|h_{l}(N \beta)\right|$, we may employ Debye's and Watson's asymptotic expansions ${ }^{19}$ ). Let $l^{\prime}<N \beta$ and $l^{\prime \prime}>N \beta$ be values of $l$ such that the Debye expansions may be employed for $l \leqslant l^{\prime}$ or $l \geqslant l^{\prime \prime}$. We have, approximately,

$$
l^{\prime} \approx N \beta-5(N \beta)^{1 / 3} ; l^{\prime \prime} \approx N \beta+5(N \beta)^{1 / 3} .
$$

It is found that

$$
\begin{gathered}
\left|h_{l}(N \beta)\right| \approx(N \beta)^{-1}\left[1-\left(\frac{2 l+1}{2 N \beta}\right)^{2}\right]^{-1 / 4} \text { for } 0 \leqslant l \leqslant l^{\prime}, \\
\left|h_{l}(N \beta)\right|=O\left[(N \beta)^{-5 / 6}\right] \text { for } l^{\prime}+1 \leqslant l \leqslant l^{\prime \prime}-1, \\
\left|h_{l}(N \beta)\right|<(N \beta)^{-5 / 6}\left(\frac{2 l+1}{2 N \beta}\right)^{l} \text { for } l \geqslant l^{\prime \prime} .
\end{gathered}
$$

Let us decompose the second member of (B l) into three parts: the sum from 0 to $l^{\prime}\left(U_{1}\right)$, the sum from $l^{\prime}+1$ to $l^{\prime \prime}-1\left(U_{2}\right)$, and the sum from $l^{\prime \prime}$ to $\infty\left(U_{3}\right)$. In $U_{1}$ and in $U_{2}$, we employ the inequality: $\left|S_{l}(k)-1\right| \leqslant 2$; in $U_{3}$, we employ the inequality (20). It then follows from (B 1) to (B 5) that

$$
\begin{gathered}
U_{1} \leqslant(N \beta)^{-1} \sum_{l=0}^{l^{\prime}}(2 l+1)\left[1-\left(\frac{2 l+1}{2 N \beta}\right)^{2}\right]^{-1 / 4}=\frac{4}{3} N \beta+O\left(\beta^{1 / 2}\right), \\
U_{2} \leqslant\left(l^{\prime \prime}-l^{\prime}\right) \cdot O\left(\beta^{1 / 6}\right)=O\left(\beta^{1 / 2}\right), \\
U_{3}<\frac{e}{N}(N \beta)^{1 / 6} \sum_{l=l^{\prime \prime}}^{\infty}\left(\frac{e^{2}}{2 N^{2}}\right)^{l}=\frac{e}{N}\left[1-\left(\frac{e^{2}}{2 N^{2}}\right)\right]^{-1}(N \beta)^{1 / 6}\left(\frac{e^{2}}{2 N^{2}}\right)^{l^{\prime \prime}}
\end{gathered}
$$

Putting together these results, we obtain the inequality (36).

To prove (37), we employ the following inequality, which follows from termwise differentiation of (31) with respect to $r$ (this is justified by the uniform convergence of the derivative series):

$$
\left|\frac{\partial u_{s}}{\partial r}(k, N a, \theta)\right| \leqslant \frac{1}{2} k \sum_{0}^{\infty}(2 l+1)\left|S_{l}(k)-1\right|\left|h_{l}^{\prime}(N \beta)\right| .
$$

If we also employ the relation

$$
h_{l}^{\prime}(z)=\frac{l}{(2 l+1)} h_{l-1}(z)-\frac{(l+1)}{(2 l+1)} h_{l+1}(z)
$$

the inequality (37) can be derived by the same procedure that has been used for the derivation of (36). 


\section{REFERENCES}

1) Van Kampen, N. G., Phys. Rev. 89 (1953) 1072.

2) Van Kampen, N. G., Phys. Rev. 91 (1953) 1267; Physica 20 (1954) 115.

3) Bogoliubov, N. N. and Shirkov, D. V., Introduction to the theory of quantized fields, Interscience, New York (1959), chapter IX.

4) Khuri, N. N., Phys. Rev. 107 (1957) 1148

5) Klein, A. and Zemach, C., Annals of Physics 7 (1959) 440.

6) Khuri, N. N. and Treiman, S. B., Phys. Rev. 109 (1958) 198.

7) Titchmarsh, E. C., The theory of functions, 2nd ed., Clarendon Press, Oxford (1939), p. 95.

8) Hobson, E. W., The theory of spherical and ellipsoidal harmonics, University Press, Cambridge (1955), p. 60.

9) Schiff, L. I., Quantum mechanics, McGraw-Hill, New York, (1949), p. 107.

10) Baker, B. B. and Copson, E. T., The mathematical theory of Huygens' principle, 2nd ed., Clarendon Press, Oxford (1950), p. 26.

11) Watson, G. N., A treatise on the theory of Bessel functions, 2nd ed., University Press, Cambridge (1952), p. 378.

12) Nevanlinna, K., Eindeutige analytische Funktionen, 2.Aufl., Springer Verlag, Berlin (1953), p. 44 .

13) Bieberbach, L., Lehrbuch der Funktionentheorie, vol. 1, Chelsea Publishing Co., New York (1945), p. 172.

14) Bargmann, V., Proc. Nat. Acad. Sci. U.S. 38 (1952) 961.

15) Humblet, J., Mém. in- $8^{\circ}$ Soc. roy. Sc. Liège 12 (1952) no. 4.

16) See e.g. Landau, L. D. and Lifshitz, E. M., Quantum mechanics, Pergamon Press, London (1958) p. 404.

17) Carter, D. S., Princeton thesis (1952), unpublished (this result is quoted in reference 4).

18) Kohr, W., Rev. Mod. Phys. 26 (1954) 292.

19) Jahnke, E. and Emde, F., Tables of functions, 4th ed., Dover, New York (1945), p. 139.

20) Kear, G., Annals of Physics 6 (1959) 102.

21) Allcock, G. R., Nuclear Physics 14 (1959) 177. 